\title{
The Evolution of Neutral Gas in the Universe
}

\section{David Turnshek, ${ }^{1}$ Sandhya Rao, ${ }^{1}$ Eric Monier, ${ }^{2}$ Daniel Nestor, ${ }^{3}$ and Anna Quider ${ }^{1}$}

${ }^{1}$ University of Pittsburgh (turnshek@pitt.edu), ${ }^{2}$ SUNY Brockport, ${ }^{3}$ IoA, Cambridge University

Abstract. We give references to some of our work on the properties and evolution of the neutral gas component of the Universe (see reference list). The bulk of the observed neutral gas has been detected by identifying intervening damped Ly $\alpha$ (DLA) quasar absorption-line systems with $\mathrm{N}(\mathrm{H}) \geqslant 2 \times 10^{20}$ atoms $\mathrm{cm}^{-2}$. We also present some initial results from a program to identify DLA absorbers near redshift $z=0.5$ using Hubble Space Telescope ACS prism spectra (see Figure 1).

Keywords. quasars: absorption lines, galaxies: evolution

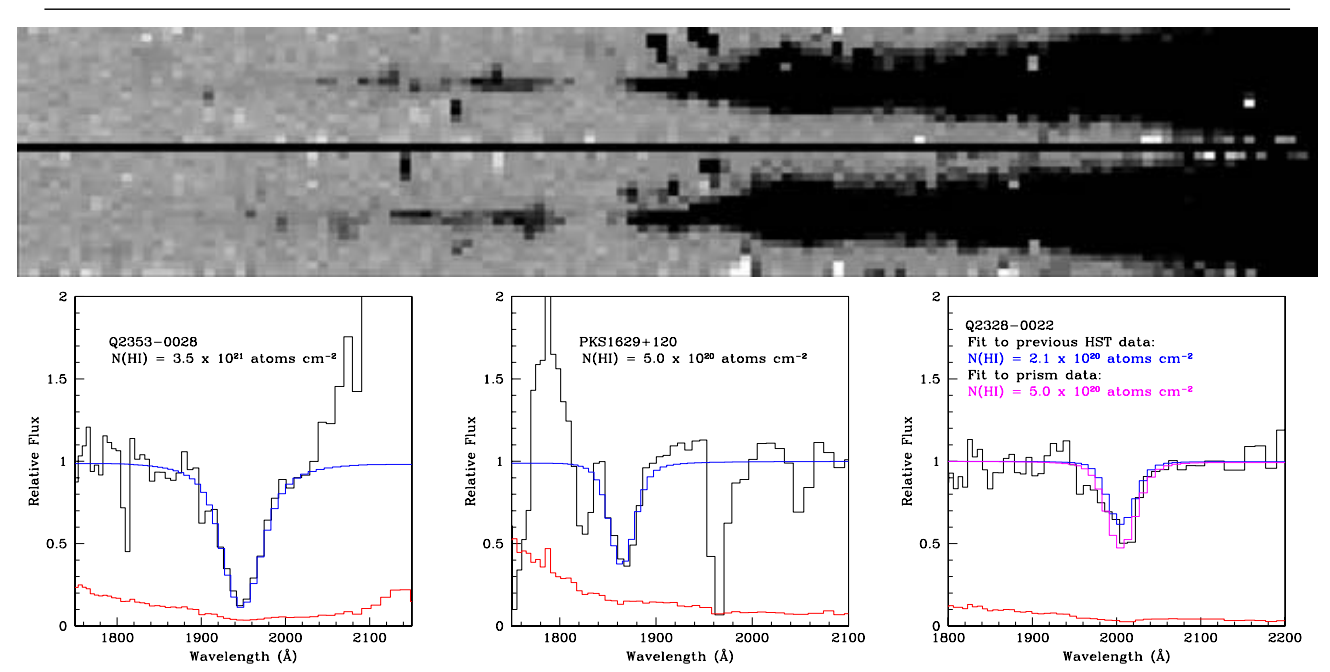

Figure 1. Results from HST ACS P200L prism observations of DLA systems previously measured using higher-resolution HST UV spectra. The top panel displays the two (dithered) prism images of the extracted high-N(HI) DLA spectrum shown in the first bottom panel. The two extracted spectra in the second and third bottom panels have lower-N(HI) DLAs. There is excellent agreement between the HST ACS prism results and the higher resolution results reported in Rao et al. (2006) for the two strongest systems, while the weaker system shows some discrepancy.

Hopkins, A, Rao, S., \& Turnshek, D. 2006, ApJ, 630, 108

Menard, B., et al. 2006, in preparation

Nestor, D., Turnshek, D., Rao, S., \& Quider, A. 2006, ApJ, in press

Rao, S., et al. 2003, ApJ, 595, 94

Rao, S., Turnshek, D., \& Nestor, D. 2006, ApJ, 636, 610

Turnshek, D., et al. 2005, IAU Symp 199 (CUP), p104 (astro-ph/0506701)

Zibetti, S., et al. 2006, astro-ph/0609760 